\title{
Dynamic expression of SMAD3 is critical in osteoblast differentiation of PDMCs
}

\author{
HSI-TING LIN ${ }^{1}$, SHAO-KUAN CHEN ${ }^{2,3}$, JIUN-WEN GUO ${ }^{4,5}$, I-CHANG SU ${ }^{6}$, \\ CHI-JUNG HUANG ${ }^{4,5,7}$, CHIH-CHENG CHIEN ${ }^{3,8}$ and CHIH-JU CHANG ${ }^{3,9}$
}

${ }^{1}$ Department of Orthopedics, Cathay General Hospital, Taipei 10630; ${ }^{2}$ Department of Urology, Sijhih Cathay General Hospital, New Taipei City 22174; ${ }^{3}$ School of Medicine, College of Medicine, Fu Jen Catholic University, New Taipei City 24205; ${ }^{4} \mathrm{Ph}$.D. Program in Pharmaceutical Biotechnology, College of Medicine, Fu Jen Catholic University, New Taipei City 24205;

${ }^{5}$ Department of Medical Research, Cathay General Hospital, Taipei 10630; ${ }^{6}$ Department of Neurosurgery, Sijhih Cathay General Hospital, New Taipei City 22174; ${ }^{7}$ Department of Biochemistry, National Defense Medical Center, Taipei 11490; Departments of ${ }^{8}$ Anesthesiology and ${ }^{9}$ Neurosurgery, Cathay General Hospital, Taipei 10630, Taiwan, R.O.C.

Received July 27, 2018; Accepted November 19, 2018

DOI: $10.3892 / \mathrm{ijmm} .2018 .4001$

\begin{abstract}
Human pluripotent stem cells have the potential assist in the identification of genes involved in mammalian development. The human placenta is considered a repository of stem cells, termed placenta-derived multipotent cells (PDMCs), which are able to differentiate into cells with an osteoblastic phenotype. This plasticity of PDMCs maybe applied clinically to the understanding of osteogenesis and osteoporosis. In the presentstudy, osteoblasts were generated by culturing PDMCs in osteogenic medium. Reverse transcription quantitative polymerase chain reactionand the degree of osteoblast calcification were used to evaluate the efficacy of osteogenesis. The results suggestedthat the expression of mothers against decapentaplegic homolog 3 (SMAD3) increased in the initial stages of osteogenic differentiation but decreased in the later stages. However, osteogenesis was inhibitedwhen the PDMCs overexpressed SMAD3 throughout the differentiation period. In addition, the rate of osteogenic differentiation was decreased when SMAD3 signaling was impaired. In conclusion, SMAD3 serves an important role in osteoblast differentiation and bone formation in a time-dependent manner. The data from the present study indicate that arapid increase in SMAD3 expression is crucial for osteogenesis and suggest a role for PDMCs in the treatment of patients with osteoporosis.
\end{abstract}

Correspondence to: Dr Chih-Ju Chang, Department of Neurosurgery, Cathay General Hospital, 280 Ren'ai Rd., Sec. 4, Taipei 10630, Taiwan, R.O.C.

E-mail: miklechang5639@gmail.com

Key words: placenta-derived multipotent cells, osteogenesis, osteoporosis, osteoblast, mothers against decapentaplegic homolog 3

\section{Introduction}

Human pluripotent stem cells have the potential to assist in the identification of genes associated with mammalian development $(1,2)$, and the plasticity of stem cells suggests that they may have a wide range of clinical applications $(3,4)$. Bone tissue engineering has also been explored for clinical purposes $(5,6)$.

A number of growth factors, cytokines, drugs and gene products are critical for the differentiation of stem cells, including osteoblast differentiation (7-9). Indeed, one of the pivotal factors in the health and maintenance of bone density is the coordinated activity of osteoblasts and osteoclasts (10). For example, bone loss in older people due to imbalances between osteoblast and osteoclast activitymay servea role in post-fracture mortality $(11,12)$. In addition, appropriate osteoblast calcification or bone mineralization is essential for complete bone formation (13-15). The process of bone regeneration has the potential to treat defects in bone healing in patients with osteoporosis with bone fractures or abnormalities in osteogenesis $(9,16,17)$.

Osteoporosis is a musculoskeletal disease characterized by decreasedbone mineral density and an increased risk of fragility fractures (18). It is known that in the bones of patients with osteoporosis, resorption by osteoclasts exceeds bone formation by osteoblasts (19). Clinically, osteoporotic fractures result in notable mortality and morbidity (18). Alendronate acid, a bisphosphonate, is the primary agent used to prevent and treat osteoporosis (20-22). Pharmacokinetic studies have indicated that $\sim 50 \%$ of the alendronate absorbed into the body binds to bone surface, while the other $50 \%$ is rapidly excreted from the kidneys $(20,23,24)$. Besides conventional chemical therapy, stem cell therapy has been demonstrated to lead to an improvement in patients with osteoporosis by promoting osteogenesis and preventing the progression of osteoporosis $(25,26)$. We previously isolated a population of multipotent cells from a human full-term placenta, termed placenta-derived multipotent cells (PDMCs), which were able to differentiate into a range of cell types, including cells with an osteoblastic phenotype (27). 
In addition, human PDMCs have demonstrated marked therapeutic effects in animal models following their engraftment within a lesion (28). Therefore, understanding the molecular mechanism of the differentiation of PDMCs into osteoblasts may assist in treating patients with osteoporosis.

The present study aimed to identify molecules that were differentially expressed during osteogenic development. Firstly, the significant genes were identified from a microarray dataset in the gene expression omnibus (GEO) repository. Osteoblasts were generated by culturing PDMCs in osteogenic medium (OM) for different times. Reverse transcription quantitative polymerase chain reaction (RT-qPCR) was performed and osteoblast calcification was measured to evaluate the efficacy of osteogenesis.

\section{Materials and methods}

Isolation and phenotypic characterization of PDMCs. A full-term placenta (38-40 weeks gestation) from a healthy 27-year-old woman was obtained under a procedure approved by the Institutional Review Board of Cathay General Hospital (Taipei, Taiwan; approval no. CT98115). The individual provided informed consent. The stem cells (PDMCs) were isolated as described previously $(7,27)$. Prior to any differentiation, a minimal immunophenotyping was performed as previously described, with minor modifications of the antibody titers as described, to validate the stemness of the PDMCs using a FACSCalibur flow cytometer and CellQuest software (v.3.3; both BD Biosciences, Franklin Lakes, NJ, USA) (29,30). Briefly, 'stemness' was defined as cells within the PDMC population predominantly staining for integrin $\beta 1$ (cat. no. 303003; BioLegend, San Diego, CA, USA) (1:50), cluster of differentiation (CD)44 (cat. no. 555478) (1:50), 5'-nucleotidase (cat. no. 550256) (1:50), Thy-1 membrane glycoprotein (cat. no. 555595; all BD Biosciences) (1:50), endoglin (cat. no. MAB2152; Chemicon, EMD Millipore, Billerica, MA, USA) (1:50), and CD166 (cat. no. MCA 1926F; AbDSerotec, Bio-Rad Laboratories, Inc., Hercules, CA, USA) (1:5) and lacking expression of human leukocyte antigen-DR isotype (cat. no. 555818;) (Class II, 1:50), hematopoietic progenitor cell antigen CD34 (cat. no. 341071; both $\mathrm{BD}$ Biosciences) and receptor-type tyrosine-protein phosphatase $\mathrm{C}(1: 50)$, and monocyte differentiation antigen CD14 (cat. no. 301805; BioLegend) $(1: 50)(31,32)$.

Osteoblast differentiation. Induced osteoblast differentiation was achieved as described previously, with certainminor modifications (27,33). Briefly, PDMCs were maintained in a basal medium, and osteogenic differentiation was induced by culture in $\mathrm{OM}$, which consisted of basal medium containing $0.1 \mu \mathrm{M}$ dexamethasone, $10 \mathrm{mM} \beta$-glycerol phosphate, and $50 \mu \mathrm{M}$ sodium L-ascorbate (all Sigma-Aldrich; Merck KGaA, Darmstadt, Germany), at a density of 17,000 cells $/ \mathrm{cm}^{2}$. The differentiated cells were then harvested for subsequent analysis.

Expression profile data. Data in GSE35959, deposited by Benisch et al (34) was downloaded from the Gene Expression Omnibus (GEO: www.ncbi.nlm.nih.gov/geo/) database. A total of two groups (Group 1:4 controls without osteoporosis; 1 male and 3 females; age range: $79-89$ years and Group 2: 5 patients with osteoporosis; 5 females; age range: $79-94$ years) were enrolled to study the differentially expressed genes.
Validation of gene expression and RT-qPCR. To determine gene expression levels, total RNA was extracted from cells at each experimental time point using TRIzol ${ }^{\circledR}$ reagent (Thermo Fisher Scientific, Inc., Waltham, MA, USA). Total RNA $(1 \mu \mathrm{g})$ was reverse-transcribed using oligo $(\mathrm{dT})_{20}$ primer and SuperScript III First-Strand Synthesis System (Invitrogen; Thermo Fisher Scientific, Inc.) according to the manufacturer's protocol. The relative mRNA level of the target gene was quantified using qPCR with a TaqMan probe and TaqMan Master Mix in a LightCycler Nano instrument (both Roche Diagnostics GmbH, Mannheim, Germany), according to the manufacturer's protocol. Amplification primers and specific TaqMan probes for candidate genes were designed by Roche Diagnostics GmbH, and are summarized in Table I. Expression of each gene was normalized to that of GAPDH to avoid errors due to sample-to-sample differences in RNA quantity. Light Cycler Software (version 1.1.0; Roche Diagnostics GmbH) was used to analyze the PCR kinetics and to calculate quantitative results. The two-step qPCR conditions were as follows: Initial denaturation at $95^{\circ} \mathrm{C}$ for $2 \mathrm{~min}$, then 50 cycles of amplification (denaturation at $95^{\circ} \mathrm{C}$ for $20 \mathrm{sec}$; annealing and elongation at $60^{\circ} \mathrm{C}$ for $20 \mathrm{sec}$ ). The data were analyzed using the $2^{-\Delta \Delta \mathrm{Cq}}$ method, and results are expressed as the ratio of expression at the designated time compared with that at time 0 (35).

Mothers against decapentaplegic homolog 3 (SMAD3) overexpression. To produce tetracycline-inducible SMAD3, the cDNA encoding SMAD3 was prepared by amplification with forward primer (5'-GCTAGCATGTCGTCCATC CTGCCTTT-3') and reverse primer (5'-GATATCAGACAC ACTGGAACAGCGG-3') from a human placenta library (Sigma-Aldrich; Merck $\mathrm{KGaA}$ ) and then sequenced to confirm gene identity. SMAD3-overexpressing PDMCs (over SMAD3-PDMCs) were generated by lentiviral transduction using the all-in-one tetracycline-inducible plasmid (pAS4.1w. Ppuro-aOn). This doxycycline-induced SMAD3 contained 3 extra amino acids at the C-terminus of SMAD3 due to the cloning strategy. Infection of each lentivirus into PDMCs was performed according to the protocol used in the National RNAi Core Facility at the Institute of Molecular Biology/Genomic Research Center, Academia Sinica (Taipei, Taiwan) (36). Briefly, $1.5 \times 10^{6}$ cells were grown in a $10 \mathrm{~cm}$ dish for $24 \mathrm{~h}$, and a multiplicity of infection of 3 was used for subsequent lentiviral infections. In the presentstudy, all plasmids and the viral particles generated were purchased from the National RNAi Core Facility. Firstly, the over SMAD3-PDMCs were used to elucidate the molecular effects of SMAD3 on osteogenic differentiation. Conditional SMAD3 expression in over SMAD3-PDMCs was induced by adding doxycycline ( $1 \mu \mathrm{g} / \mathrm{ml}$ ) to cause overexpression of SMAD3 3 days prior, on the day, 3 days following, and 6 days following OM treatment for osteogenic differentiation. These cells were lysed and harvested using radioimmunoprecipitation assay lysisbuffer containing Protease Inhibitor Cocktail (both Sigma-Aldrich; Merck KGaA) according to the manufacturer's protocol. The protein aliquot $(20 \mu \mathrm{g})$ of each lysate, quantified using Bradford protein assay (Bio Rad Laboratories, Inc.), was separated by the NuPAGE 4-12\% Bis-Tris Gel (Thermo Fisher Scientific, Inc.) and transferred onto a polyvinylidene fluoride membrane (Merck KGaA) using a TE70 Semi-Dry Transfer 
Table I. Amplification primers and specific TaqMan probes for candidate genes.

\begin{tabular}{|c|c|c|c|}
\hline Gene name & Accession no. & Sequence (5' to $3^{\prime}$ ) & UPL probe no. \\
\hline GAPDH & NM_002046.3 & $\begin{array}{l}\text { F: CTCTGCTCCTCCTGTTCGAC } \\
\text { R: ACGACCAAATCCGTTGACTC }\end{array}$ & 60 \\
\hline ALP & NM_001127501.1 & $\begin{array}{l}\text { F: AGAACCCCAAAGGCTTCTTC } \\
\text { R: CCTGCTTGGCTTTTCCTTC }\end{array}$ & 12 \\
\hline BMP-2 & NM_001200.2 & $\begin{array}{l}\text { F: CAGACCACCGGTTGGAGA } \\
\text { R: CCCACTCGTTTCTGGTAGTTCT }\end{array}$ & 31 \\
\hline COL1A1 & NM_000088.3 & $\begin{array}{l}\text { F: GGGATTCCCTGGACCTAAAG } \\
\text { R: GGAACACCTCGCTCTCCA }\end{array}$ & 67 \\
\hline RUNX2 & NM_004348.3 & $\begin{array}{l}\text { F: GTGCCTAGGCGCATTTCA } \\
\text { R: GCTCTTCTTACTGAGAGTGGAAGG }\end{array}$ & 29 \\
\hline SMAD3 & NM_005902.3 & $\begin{array}{l}\text { F: GTCTGCAAGATCCCACCAG } \\
\text { R: AGCCCTGGTTGACCGACT }\end{array}$ & 79 \\
\hline
\end{tabular}

GADPH, Glyceraldehyde 3-phosphate dehydrogenase; ALP, Alkaline phosphatase; BMP-2, Bone morphogenetic protein 2; COL1A1, Collagen, type I, $\alpha 1$ chain; RUNX2, Runt-related transcription factor 2; SMAD3, mothers against decapentaplegic homolog 3; UPL, universal probe library; $\mathrm{F}$, forward primer; $\mathrm{R}$, reverse primer.

Unit (GE Healthcare, Chicago, IL, USA). This membrane was then blocked in $3 \%$ bovine serum albumin (cat. no. A3803; Sigma-Aldrich; Merck KGaA) and $10 \%$ non-fat milk for $30 \mathrm{~min}$ at room temperature, and SMAD3 protein levels were detected with anti-SMAD3 antibodies (cat. no. ab40854; 1:5,000; Abcam, Cambridge, MA, USA) for $30 \mathrm{~min}$. The protein level of GAPDH was treated as a protein-loading control to ensure equal protein levels in all the samples using an anti-GAPDH antibody (cat. no. AM4300; dilution; 1:6,000; Thermo Fisher Scientific, Inc.). The secondary antibodies were biotinylated anti-rabbit anti-SMAD3 IgG (cat. no. BA-1000; cat. no. 1:1,000; Vector Laboratories, Burlingame, CA, USA) and horseradish peroxidase-conjugated anti-mouse anti-GAPDH IgG (cat. no. ab6808; cat. no. 1:5,000; Abcam). The bands were then visualized using the VECTASTAIN ABC-AmP Chemiluminescence Detection kit (cat. no. AK-6601; Vector Laboratories) for the SMAD3 protein and the Western Lightning Ultra-ECL (PerkinElmer, Waltham, MA, USA) for the GAPDH protein, according to the manufacturers' protocol. Finally, images were captured using an Alpha InnotechFluorChem FC2 Imager (Alpha Innotech; ProteinSimple, San Jose, CA, USA).

Inhibition of SMAD3 signaling. The SMAD3 inhibitor, SIS3 (cat. no. 1009104-85-1; Cayman Chemical Company, Ann Arbor, MI, USA), was applied to inhibit SMAD3 signaling. Briefly, different concentrations of $\operatorname{SIS} 3(0,1,3$ and $10 \mu \mathrm{M})$ in combination with doxycycline $(1 \mu \mathrm{g} / \mathrm{ml})$ were added to the over SMAD3-PDMCs for 3 days prior to the addition of OM to induce osteogenic differentiation.

Alizarin red staining $(A R S)$. Staining with alizarin red, an anthraquinone dye that indicates calcium deposition, was performed to evaluate the efficacy of osteogenic differentiation. Briefly, the cells were fixed in $70 \%$ ethanol at $4^{\circ} \mathrm{C}$ for $1 \mathrm{~h}$, then washed with PBS. Next, the cells were incubated with staining solution (40 $\mathrm{mM}$ alizarin red $\mathrm{S}$; $\mathrm{pH} 4.2$; cat. no. A5533; Sigma-Aldrich; Merck KGaA) at room temperature for $5 \mathrm{~min}$ and then washed 3 times with distilled water. Images were captured using an Olympus IX70 microscope with the magnification x100 (Olympus Corporation, Tokyo, Japan) and Nikon COOLPIX5000 (Nikon Corporation, Tokyo, Japan). The ARS signals were identified as regions of red or reddish color, and were calculated as the ratios in the regions of interest (ROIs) using CellCloud software (v.0.8; Meridigen Biotechnology, Taipei, Taiwan). The positive signals in each raw image were calculated using superpixels, segmentation and object identification. Briefly, the analysis module firstly used a superpixel algorithm to partition the image into multiple segments, using a set of parameters to identify the ROIs in each raw image that exhibited positive ARS, and then calculate the ratio of the ROIs.

Calcium colorimetric assay. Calcification was assessed using the calcium O-cresolphthaleincomplexone method (37). Briefly, the cell medium at each experimental time point was removed and cells were washed twice with PBS. The cells were mixed with $0.5 \mathrm{M}$ acetic acid for $20 \mathrm{~min}$ at room temperature. The samples $(10 \mu \mathrm{l})$ were transferred into a microtiter plate, and $200 \mu 1$ 2-amino-2-methyl-1-propanol solution (Sigma-Aldrich; Merck KGaA) and $200 \mu \mathrm{l}$ O-cresolphthaleincomplexone reagent (Sigma-Aldrich; Merck KGaA) were added. The plates were incubated at $25^{\circ} \mathrm{C}$ for $15 \mathrm{~min}$ and then the absorbance at $575 \mathrm{~nm}$ was determined using a Synergy HT Microplate Reader (BioTek Instruments, Inc., Winooski, VT, USA).

Statistical analysis. All values were obtained from independently performed duplicate or triplicate experiments. Data are presented as the mean \pm standard deviation. Statistical comparisons were conducted using One-way analysis of variance followed by a post-hoc test using Bonferroni correction. The unpaired t-test was used to analyze two 

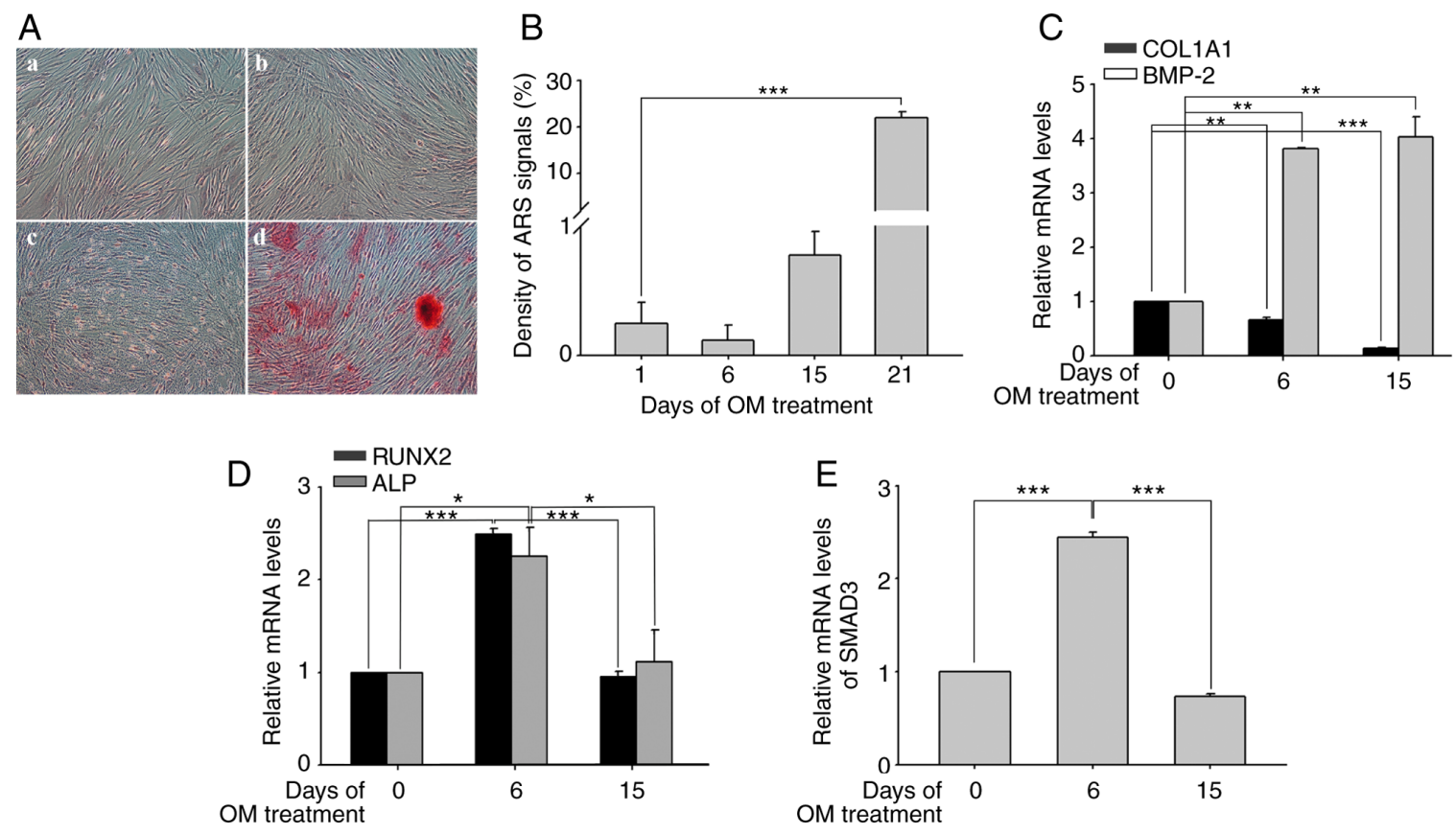

Figure 1. Efficacy results of osteogenic differentiation and relative gene expression levels under OM treatment. (A) The ARS signals in OM-treated PDMCs. ARS signals were acquired from cells following OM treatment at different days. (A-a) Day 1. (A-b) Day 6. (A-c) Day 15. (A-d) Day 21. (B) The relative intensity of the ARS signals in OM-treated PDMCs. (C) Expression levels of target osteogenic genes RUNX2 and ALP. (D) Expression levels of target osteogenic genes COL1A1 and BMP-2; (E) SMAD3 gene expression levels. All gene expressions were compared with that of GAPDH. The ARS signals were calculated as the ratios in the regions of interest using CellCloud software. Statistical analysis was performed by one-way analysis of variance. ${ }^{*} \mathrm{P}<0.05,{ }^{* * *} \mathrm{P}<0.01$ and ${ }^{* * *} \mathrm{P}<0.001$. ARS, alizarin red staining; OM, osteogenic medium; PDMCs, placenta-derived multipotent cells; SMAD3, mothers against decapentaplegic homolog 3; RUNX2, Runt-related transcription factor 2; ALP, alkaline phosphatase; COL1A1, Collagen, type I, alpha 1 chain; BMP-2, Bone morphogenetic protein 2.

independent groups. Statistical analyses were performed using IBM SPSS v. 22.0 software (IBM Corp., Armonk, NY, USA). $\mathrm{P}<0.05$ was considered to indicate a statistically significant difference.

\section{Results}

Changes in ARS signals in OM-treated PDMCs. The ARS signals in OM-treated PDMCs increased in strength in a time-dependent manner (Fig. 1A). The relative intensity of the ARS signals was quantified, and it was demonstrated that PDMCs with calcium deposition $(0.78 \pm 0.13 \%)$ were identified at day 15 of $\mathrm{OM}$ treatment and intense calcium deposition was detected on day $21(21.89 \pm 1.01 \%$; Fig. 1B). Using a database (GSE35959 for osteoporosis) from the Gene Expression Omnibus repository, it was identified that SMAD3 expression was upregulated $\left(\log _{2}\right.$ ratio=5.14) in the mesenchymal stem cells of patients with primary osteoporosis compared with that in individual controls (Table II). Therefore, SMAD3 was selected for additional analysis due to its significance in bone mineralization and osteoblast differentiation $(38,39)$.

Quantification of osteogenesis-associated genes and SMAD3 in OM-treated PDMCs. The mRNA levels of several other osteogenesis-associated genes and SMAD3 in OM-treated PDMCs compared with the levels in cells on day 0 of osteogenic differentiation were quantified. Firstly, the expression of bone morphogenic protein 2 (BMP-2), an important factor in osteogenesis, was increased in OM-treated PDMCs on day 6 (3.81) and at day 15 (4.02). By contrast, collagen type alpha 1 chain (COL1A1), a marker of chondrogenesis, was downregulated after $6(0.62)$ and 15 (0.13) days in OM (Fig. 1C). The changes in expression of two molecules closely associated with the osteoblast phenotype, runt-related transcription factor 2 (RUNX2) and alkaline phosphatase (ALP) (Fig. 1D) were also quantified. RUNX2 and ALP were induced at day $6($ RUNX2=2.45; ALP=1.93), but decreased at day 15 (RUNX2=0.91; ALP=1.10); this expression pattern was similar to that observed for SMAD3. As indicated in Fig. 1E, SMAD3 expression increased to 2.39 at day 6 of OM treatment and then markedly decreased to 0.76 at day 15 .

Role of SMAD3 in mineralization during osteogenesis. In an attempt to understand the role of SMAD3 in mineralization during osteogenesis, over SMAD3-PDMCs that overexpressed SMAD3 via a doxycycline-inducible system were constructed. Fig. 2A describes the experimental design, indicating the times of doxycycline induction, osteogenic differentiation by $\mathrm{OM}$, and analyses following harvesting of the cells. Fig. 2B demonstrates that there were increases of up to 10 -fold in the mRNA expression of SMAD3 in over SMAD3-PDMCs induced by doxycycline prior to OM treatment compared with that in the non-induced cells. When the calcium deposition was analyzed by ARS, the red signal intensity reached a peak at day 21 following OM treatment (Fig. 2C). Quantification of the ARS signal and the intracellular level of calcium ions indicated a markedly high production of calcium at day 21 [ARS signal=86.03 $\pm 2.14 \%$ (Fig. 2D); intracellular calcium=18.49 $\pm 0.17 \mathrm{mg} / \mathrm{dl}$ (Fig. 2E)]. However, the over SMAD3-PDMCs also exhibited early deposition of calcium (ARS signal $=53.96 \pm 7.42 \%$; Fig. 2D) and a higher level of intracellular calcium $(18.10 \pm 0.29 \mathrm{mg} / \mathrm{dl}$; Fig. $2 \mathrm{E})$ at day 9 following 
Table II. Top 10 significant genes with differential expression in patients with osteoporosis.

\begin{tabular}{llclr}
\hline Gene name & Accession no. & $\log _{2}$ ratio & Regulation (up or down) & P-value \\
\hline SMAD3 & NM_005902.3 & 5.14 & Up & 0.000079 \\
COL1A1 & NM_000088.3 & 4.22 & Up & 0.000007 \\
IGF2 & NM_000612 & 4.18 & Up & 0.015652 \\
TGFB2 & NM_003238 & 3.93 & Up & 0.004562 \\
FGFR1 & NM_015850 & 3.65 & Up & 0.000044 \\
COL14A1 & NM_021110 & -3.789 & Down \\
FGFR2 & NM_000141 & Down & 0.012965 \\
NOG & NM_005450 & -3.042 & Down & 0.002195 \\
CDH11 & NM_001797 & -2.355 & Down & 0.016054 \\
SMAD2 & NM_005901 & -2.210 & Down & 0.024306 \\
\hline
\end{tabular}

Data was extracted from the GSE35959 dataset including two groups (Group 1: 4 controls without osteoporosis; 1 male and 3 females; age range, 79-89 years. Group 2: 5 patients with osteoporosis; 5 females; age range, 79 -94 years). SMAD3, mothers against decapentaplegic homolog 3; COL1A1, Collagen, type I, $\alpha 1$ chain; IGF2, Insulin like growth factor 2; TGFB2, Transforming growth factor $\beta 2$; FGFR1, Fibroblast growth factor receptor 1; COL14A1, Collagen type XIV $\alpha 1$ Chain; FGFR2, Fibroblast growth factor receptor 2; NOG, Noggin; CDH11, Cadherin 11;

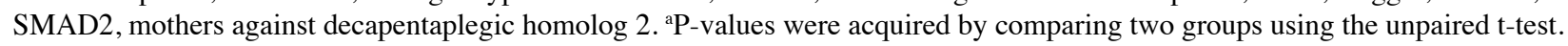
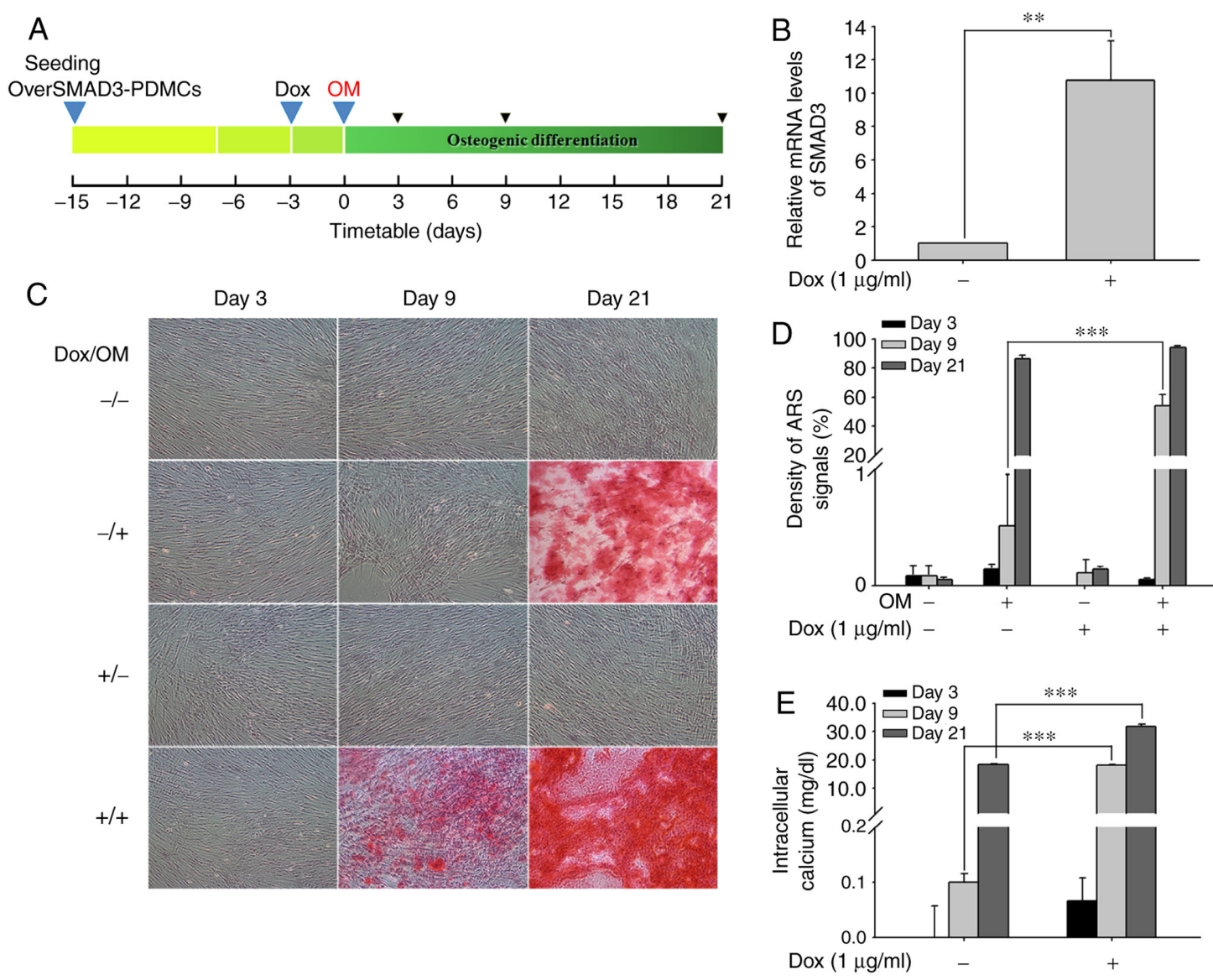

Figure 2. Effect of SMAD3 overexpression in mineralization during osteogenesis via a doxycycline-inducible system. (A) The experimental design. (B) SMAD3 mRNA expression. (C) The ARS signal intensity following OM treatment. (D) Quantification of the ARS signal. (E) The intracellular level of calcium ions. The ARS signals were calculated as the ratios in the regions of interest using CellCloud software. The statistic was calculated by one-way analysis of variance for ARS signals and intracellular calcium and the unpaired t-test for SMAD3 expressions, ${ }^{* *} \mathrm{P}<0.01$ and ${ }^{* * *} \mathrm{P}<0.001$. SMAD3, mothers against decapentaplegic homolog 3; OM, osteogenic medium; Dox, doxycycline (1 $\mu \mathrm{g} / \mathrm{ml})$; ARS, alizarin red staining.

OM treatment, and the ARS signaling (94.34 $\pm 1.11 \%$; Fig. 2D) and intracellular calcium levels $(31.88 \pm 0.77 \mathrm{mg} / \mathrm{dl}$; Fig. 2E) were additionally increased at day 21 . By contrast, the positive ARS signals were delayed and inhibited if the expression of 


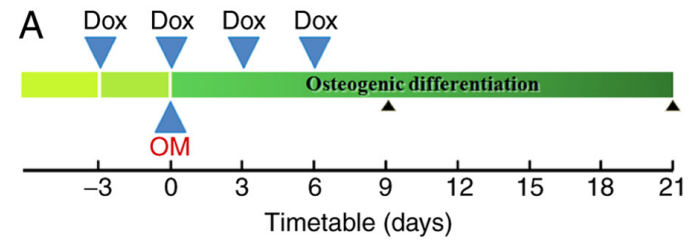

C

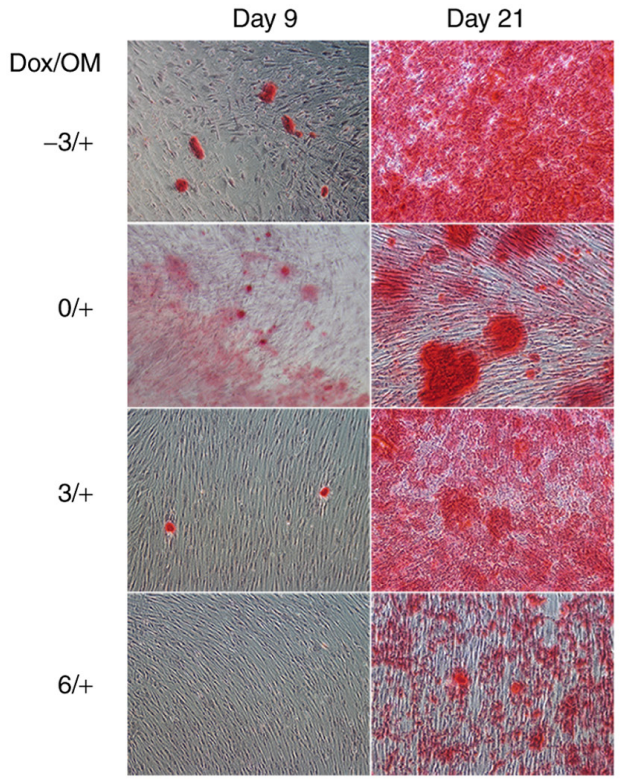

B
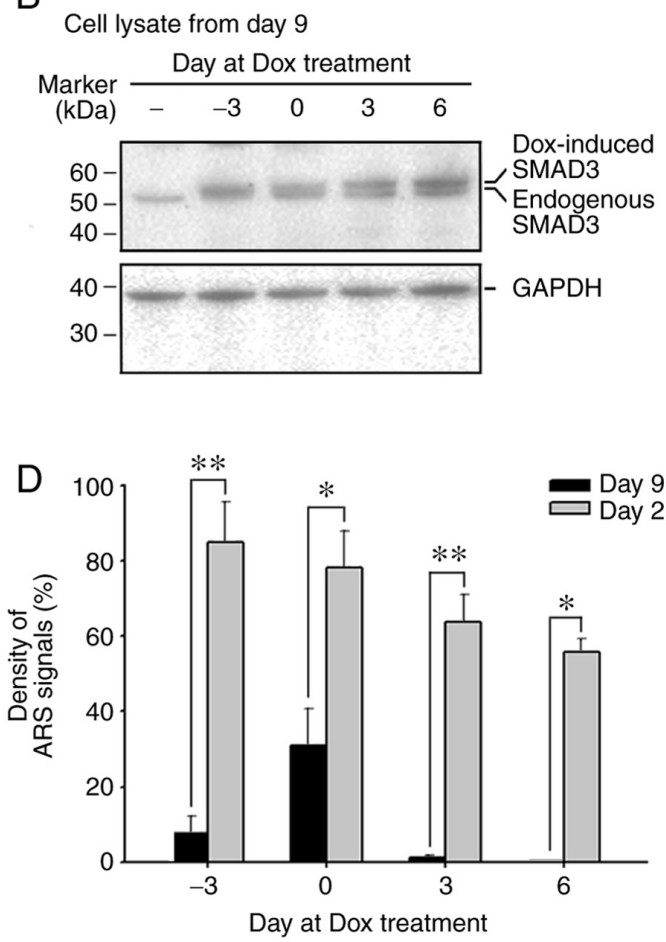

Figure 3. Expression of SMAD3 is induced by doxycycline following the initiation of OM treatment. (A) The experimental design. (B) Western blot analysis for SMAD3. (C) Changes in ARS signals and (D) quantification of ARS signals. The ARS signals were calculated as the ratios in the regions of interest using CellCloud software. Statistical analysis was performed by one-way analysis of variance. ${ }^{*} \mathrm{P}<0.05$ and ${ }^{* *} \mathrm{P}<0.01$. SMAD3, mothers against decapentaplegic homolog 3; OM, osteogenic medium; Dox, doxycycline ( $1 \mu \mathrm{g} / \mathrm{ml})$; ARS, alizarin red staining; -3, induction of SMAD3 expression 3 days prior to OM treatment; 0 , induction of SMAD3 expression on the same day of OM treatment; 3, induction of SMAD3 expression 3 days after OM treatment; 6, induction of SMAD3 expression 6 days following OM treatment.

SMAD3 was induced by doxycycline following the initiation of OM treatment, as demonstrated in Fig. 3A. The increased doxycycline-induced SMAD3 was revealed on different days using western blot analysis (Fig. 3B). A decreased ARS signal was observed (Fig. 3C) and quantified (Fig. 3D). Briefly, decreased ARS signals were detected in the cells with doxycycline induction 6 days following OM treatment (day 9, $0.02 \pm 0.02 \%$; day $21,55.63 \pm 3.28 \%$ ) compared with the cells with doxycycline induction 3 days following OM treatment $(1.20 \pm 0.48 \%$ at day 9 and $63.84 \pm 10.48 \%$ at day 21$)$.

Impairment of ARS signals in the presence of SMAD3 inhibitor SIS3. The data indicated the molecular significance of SMAD3 in the initial stages of osteoblast differentiation, so the SMAD3 inhibitor, SIS3 (CAS 1009104-85-1), was used to selectively inhibit SMAD3-mediated signaling (40). As indicated in Fig. 4, the positive ARS signal in the over SMAD3-PDMCs decreased with increasing levels of SMAD3 inhibitor. Over SMAD3-PDMCs that were not induced by doxycycline exhibited a low ARS signal $(0.05 \pm 0.03 \%)$, but those induced by doxycycline exhibited a high ARS signal $(91.56 \pm 2.70 \%)$ on day 9 following OM treatment (Fig. 4A). However, this increased ARS signal decreased when the over SMAD3-PDMCs were also treated with SIS3. Decreasing ARS signals were detected in the presence of increasing concentrations of SIS3 (from 1-10 $\mu \mathrm{M}$ ) at day 21. As indicated in Fig. 4B, the lowest ARS signal at day $21(7.11 \pm 4.44 \%)$ was obtained in the over SMAD3-PDMCs treated with the highest SMAD3 inhibitor concentration $(10 \mu \mathrm{M})$.

\section{Discussion}

Osteoporosis is a disease of the bones, in which the rate of bone resorption by osteoclasts exceeds the rate of bone formation by osteoblasts (41). The therapeutic strategy for treating patients with osteoporosis is straightforward: Either decrease the activity of osteoclasts with bisphosphonates and estrogen-associated compounds or increase the activity of osteoblasts with parathyroid hormones (42). Therefore, the agent alendronate acid is widely used to increase bone marrow density in clinics $(43,44)$.

In the presentstudy, it was demonstrated that PDMCs, like other stem cell lineages, may undergo osteogenic differentiation when cultured in OM (5). However, PDMCs are markedly different from other stem cell lineages as they are abundant, the harvesting procedure is non-invasive and they do not cause safety concerns $(29,45)$. We previously demonstrated that PDMCs may differentiate into osteoblasts through a different process to other stem cells (27). The present study demonstrated that, like osteoblasts derived from human mesenchymal stem cells $(46,47)$, OM-treated PDMCs expressed BMP-2. BMPs are known to stimulate new bone formation (48). Sun et al (46) revealed that BMP-2 may improve bone healing 


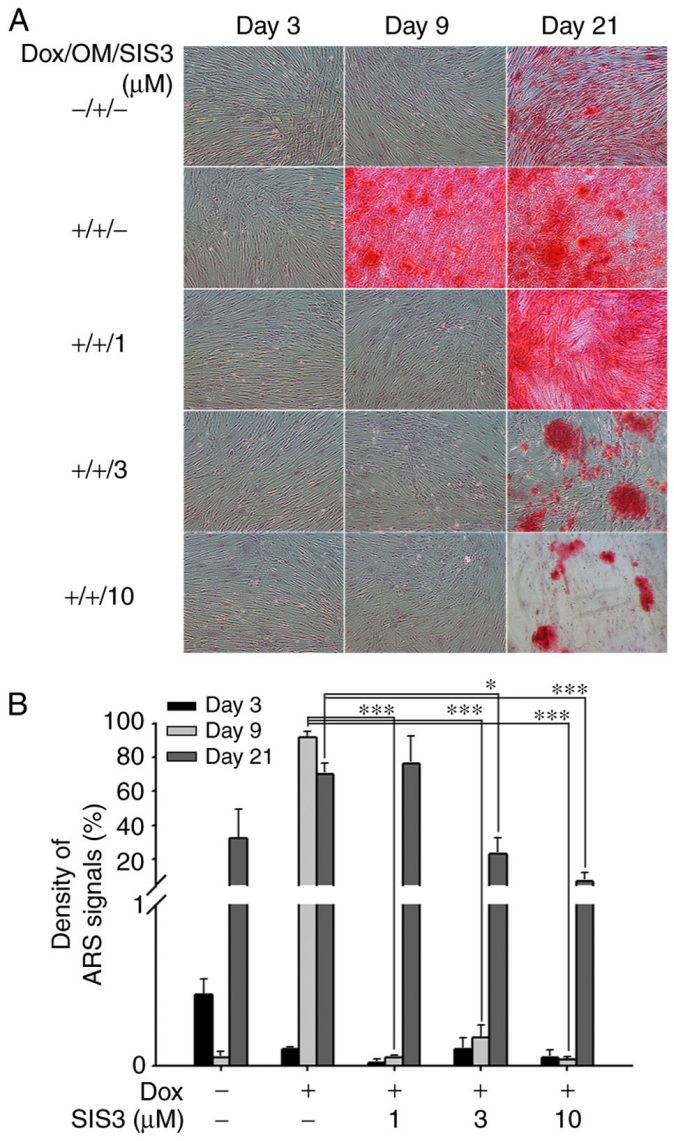

Figure 4. SMAD3 inhibitor decreases the ARS signal in the over SMAD3 placenta-derived multipotent cells undergoing osteogenic differentiation. (A) Changes in ARS signals and (B) quantification of ARS signals. The ARS signals were calculated as the ratios in the regions of interest using CellCloud software. Statistical analysis was performed by one-way analysis of variance. ${ }^{*} \mathrm{P}<0.05$ and ${ }^{* * *} \mathrm{P}<0.001$. SMAD3, mothers against decapentaplegic homolog 3; Dox, doxycycline (1 $\mu \mathrm{g} / \mathrm{ml})$; OM, osteogenic medium ARS, alizarin red staining; SIS3, SMAD3 inhibitor.

in an animal model. Furthermore, the molecules RUNX2 and ALP, which are associated with osteoblast differentiation and bone activity, were also upregulated during the initial stages of differentiation (49-51). It was also identified that these two molecules were expressed consistently and concurrently with the expression of SMAD3. RUNX2 was previously demonstrated to be upregulated by SMADs, and ALP serves a procalcification role in mineralization $(52,53)$. The early peak of ALP expression in OM-treated PDMCs observed in the present study was similar to that revealed by previous studies investigating mesenchymal stem cells (MSCs) $(53,54)$. Taken together, the results of the present study suggest that upregulation of SMAD3 may change the microenvironment of undifferentiated cells and, at least for OM-treated PDMCs, promote SMAD3-dependent osteoblast differentiation.

SMADs are a group of cytoplasmic signal transducers of transforming growth factor- $\beta$ and BMP signaling $(55,56)$. These molecules have widely recognized roles in bone formation during mammalian development (56). Therefore, SMADs may serve an integral role in the normal maintenance of bone and osteogenic differentiation. Although SMAD3 signaling is known to be involved in a number of cellular functions including fibrogenesis and wound healing (57-60), the present study clarified the significance of SMAD3 function in osteogenesis. In fact, it has been demonstrated that SMAD3 serves an important role in regulating bone formation (38). Furthermore, Borton et al (61) indicated that SMAD3-null mice had decreased bone mineral density. This may also be inferred from the data in the present study with OM-treated PDMCs in the presence of SMAD3 inhibitor. Briefly, increases in the concentration of SMAD3 inhibitor resulted in the detection of decreased ARS signals. Therefore, in light of the results from the present study, the defect in bone formation described by Borton et al (61) may have resulted from the absence of SMAD3 at the initial stages of development.

In addition, the present study identified that the pattern of expression of SMAD3 exhibited an increase during osteoblast differentiation, with early peaks and then decreasesat later stages in the process of osteogenesis of PDMCs to ARS-positive osteocytes under OM treatment. This suggested that SMAD3 may differentially affect osteoblast differentiation depending upon the differentiation stage (38). microRNA-145 was demonstrated to epi-genetically regulate osteogenesis by decreasing SMAD3 expression (62). It is reasonable to suggest that osteoblast differentiation, epigenetic regulation and SMAD3 expression are associated. Conversely, Tamai et al $(63,64)$ suggested that alendronate acid may activate SMAD3 to regulate cytokines production. It was hypothesized that the once- or twice-weekly alendronate acid administration may cause the rapid increase of SMAD3 expression and improve the osteoblast differentiation $(65,66)$. Taken together, these data imply that a therapeutic equivalent of alendronate acid may result in a rapid activation of SMAD3 expression for osteoblast differentiation. The expression of SMAD3 using the tetracycline-controlled transcriptional activation gene expression system was additionally controlled (67). The forced expression of SMAD3 following OM treatment in PDMCs decreased and delayed the ARS signals. This is consistent with other studies suggesting that SMAD3 may suppress the commitment of MSCs to osteoblasts, and that a loss of SMAD3 may promote the osteocytic development of the osteoblasts $(38,61)$.

In conclusion, the results of the present study demonstrated that PDMCs may develop into osteocytes under OM treatment and that the osteoblast differentiation of PDMCs may be associated with the dynamic expression of SMAD3. In addition, it was concluded that SMAD3 serves an important role in osteoblast differentiation and bone formation in a time-dependent manner; i.e., SMAD3 must be upregulated at the initial stage of osteogenesis and then downregulated at the calcification stage. This rapid increase in SMAD3 expression is crucial for osteogenesis.

\section{Acknowledgements}

The authors would like to thank the National RNAi Core Facility at Academia Sinica in Taiwan for providing reagents for lentiviral infection and related services.

\section{Funding}

The present study was supported by Department of Medical Research of Cathay General Hospital. 


\section{Availability of data and materials}

The datasets used and/or analyzed during the current study are available from the corresponding author on reasonable request.

\section{Authors' contributions}

HTL and SKC carried out the experiments and wrote the manuscript with support from CJC. JWG performed the statistical analyses and CCC obtained the PDMC samples. ICS and CJH helped supervise the project. HTL, SKC, JWG and CCC conceived the original idea. CJC supervised the project. All authors read and approved the final manuscript.

\section{Ethics approval and consent to participate}

The present study was approved by the Institutional Review Board of Cathay General Hospital (approval no. CT98115). The individual patient provided informed consent.

\section{Patient consent for publication}

Informed consent was gained.

\section{Competing interests}

The authors declare that they have no competing interests.

\section{References}

1. Guo G, von Meyenn F, Santos F, Chen Y, Reik W, Bertone P Smith A and Nichols J: Naive pluripotent stem cells derived directly from isolated cells of the human inner cell mass. Stem Cell Reports 6: 437-446, 2016.

2. Zhu Z and Huangfu D: Human pluripotent stem cells: An emerging model in developmental biology. Development 140: 705-717, 2013.

3. Correia C, Koshkin A, Carido M, Espinha N, Šarić T, Lima PA, Serra M and Alves PM: Effective hypothermic storage of human pluripotent stem cell-derived cardiomyocytes compatible with global distribution of cells for clinical applications and toxicology testing. Stem Cells Transl Med 5: 658-669, 2016.

4. Choudhary P, Booth H, Gutteridge A, Surmacz B, Louca I, Steer J, Kerby $\mathbf{J}$ and Whiting PJ: Directing differentiation of pluripotent stem cells toward retinal pigment epithelium lineage. Stem Cells Transl Med 6: 490-501, 2017.

5. Li P, Yang YM, Sanchez S, Cui DC, Dang RJ, Wang XY, Lin QX, Wang Y, Wang C, Chen DF, et al: Deubiquitinase MYSM1 is essential for normal bone formation and mesenchymal stem cell differentiation. Sci Rep 6: 22211, 2016.

6. Dang PN, Dwivedi N, Phillips LM, Yu X, Herberg S, Bowerman C, Solorio LD, Murphy WL and Alsberg E: Controlled dual growth factor delivery from microparticles incorporated within human bone marrow-derived mesenchymal stem cell aggregates for enhanced bone tissue engineering via endochondral ossification. Stem Cells Transl Med 5: 206-217, 2016.

7. Cheng YC, Huang CJ, Lee YJ, Tien LT, Ku WC, Chien R, Lee FK and Chien CC: Knocking down of heat-shock protein 27 directs differentiation of functional glutamatergic neurons from placenta-derived multipotent cells. Sci Rep 6: 30314, 2016.

8. Silva-Vargas V, Maldonado-Soto AR, Mizrak D, Codega P and Doetsch F: Age-dependent niche signals from the choroid plexus regulate adult neural stem cells. Cell Stem Cell 19: 643-652, 2016.

9. $\mathrm{Hu} \mathrm{K}$ and Olsen BR: Osteoblast-derived VEGF regulates osteoblast differentiation and bone formation during bone repair. J Clin Invest 126: 509-526, 2016.

10. Jones DR: A potential osteoporosis target in the FAS ligand/FAS pathway of osteoblast to osteoclast signaling. Ann Transl Med 3: $189,2015$.
11. Bliuc D, Nguyen ND, Alarkawi D, Nguyen TV, Eisman JA and Center JR: Accelerated bone loss and increased post-fracture mortality in elderly women and men. Osteoporos Int 26: 1331-1339, 2015.

12. Pacifici R: Estrogen, cytokines, and pathogenesis of postmenopausal osteoporosis. J Bone Miner Res 11: 1043-1051, 1996.

13. Tanaka SM and Tachibana K: Frequency-dependence of mechanically stimulated osteoblastic calcification in tissue-engineered bone in vitro. Ann Biomed Eng 43: 2083-2089, 2015.

14. Amin Yavari S, Chai YC, Böttger AJ, Wauthle R, Schrooten J, Weinans $\mathrm{H}$ and Zadpoor AA: Effects of anodizing parameters and heat treatment on nanotopographical features, bioactivity, and cell culture response of additively manufactured porous titanium. Mater Sci Eng C Mater Biol Appl 51: 132-138, 2015.

15. Azuma K, Shiba S,Hasegawa T, Ikeda K, Urano T, Horie-Inoue K, Ouchi Y, Amizuka N and Inoue S: Osteoblast-specific $\gamma$-glutamyl carboxylase-deficient mice display enhanced bone formation with aberrant mineralization. J Bone Miner Res 30: 1245-1254, 2015.

16. Nakamura T, Naruse M, Chiba Y, Komori T, Sasaki K, Iwamoto $\mathrm{M}$ and Fukumoto S: Novel hedgehog agonists promote osteoblast differentiation in mesenchymal stem cells. J Cell Physiol 230: 922-929, 2015.

17. Joensuu K, Uusitalo L, Alm JJ, Aro HT, Hentunen TA and Heino TJ: Enhanced osteoblastic differentiation and bone formation in co-culture of human bone marrow mesenchymal stromal cells and peripheral blood mononuclear cells with exogenous VEGF. Orthop Traumatol Surg Res 101: 381-386, 2015 .

18. Willson T, Nelson SD, Newbold J, Nelson RE and LaFleur J: The clinical epidemiology of male osteoporosis: A review of the recent literature. Clin Epidemiol 7: 65-76, 2015.

19. Canalis E: MANAGEMENT OF ENDOCRINE DISEASE: Novel anabolic treatments for osteoporosis. Eur J Endocrinol 178: R33-R44, 2018.

20. Ragsdale AB, Barringer TA III and Anastasio GD: Alendronate treatment to prevent osteoporotic fractures. Arch Fam Med 7: 583-586, 1998

21. Kanis JA, Gertz BJ, Singer F and Ortolani S: Rationale for the use of alendronate in osteoporosis. Osteoporos Int 5: 1-13, 1995

22. Drake MT, Clarke BL and Khosla S: Bisphosphonates: Mechanism of action and role in clinical practice. Mayo Clin Proc 83: 1032-1045, 2008.

23. Gertz BJ, Holland SD, Kline WF, Matuszewski BK and Porras AG: Clinical pharmacology of alendronate sodium. Osteoporos Int 3 (Suppl 3): S13-S16, 1993.

24. Porras AG, Holland SD and Gertz BJ: Pharmacokinetics of alendronate. Clin Pharmacokinet 36: 315-328, 1999.

25. Sui B, Hu C, Zhang X, Zhao P, He T, Zhou C, Qiu X, Chen N, Zhao X and Jin Y: Allogeneic mesenchymal stem cell therapy promotes osteoblastogenesis and prevents glucocorticoid-induced osteoporosis. Stem Cells Transl Med 5: 1238-1246, 2016.

26. Qi X, Zhang J, Yuan H, Xu Z, Li Q, Niu X, Hu B, Wang Y and Li X: Exosomes secreted by human-induced pluripotent stem cell-derived mesenchymal stem cells repair critical-sized bone defects through enhanced angiogenesis and osteogenesis in osteoporotic rats. Int J Biol Sci 12: 836-849, 2016.

27. Cheng CC, Chung CA, Su LC, Chien CC and Cheng YC: Osteogenic differentiation of placenta-derived multipotent cells in vitro. Taiwan J Obstet Gynecol 53: 187-192, 2014.

28. Selim AO, Selim SA, Shalaby SM, Mosaad H and Saber T: Neuroprotective effects of placenta-derived mesenchymal stromal cells in a rat model of experimental autoimmune encephalomyelitis. Cytotherapy 18: 1100-1113, 2016.

29. Chien CC, Yen BL, Lee FK, Lai TH, Chen YC, Chan SH and Huang HI: In vitro differentiation of human placenta-derived multipotent cells into hepatocyte-like cells. Stem Cells 24: 1759-1768, 2006

30. Yen BL, Huang HI, Chien CC, Jui HY, Ko BS, Yao M, Shun CT, Yen ML, Lee MC and Chen YC: Isolation of multipotent cells from human term placenta. Stem Cells 23: 3-9, 2005.

31. Lv FJ, Tuan RS, Cheung KM and Leung VY: Concise review: The surface markers and identity of human mesenchymal stem cells. Stem Cells 32: 1408-1419, 2014.

32. Ali H, Al-Yatama MK, Abu-Farha M, Behbehani K and Al Madhoun A: Multi-lineage differentiation of human umbilical cord Wharton's Jelly Mesenchymal Stromal cells mediates changes in the expression profile of stemness markers. PLoS One 10: e0122465, 2015. 
33. Wrobel E, Leszczynska J and Brzoska E: The characteristics of human bone-derived cells (HBDCS) during osteogenesis in vitro. Cell Mol Biol Lett 21: 26, 2016.

34. Benisch P, Schilling T, Klein-Hitpass L, Frey SP, Seefried L, Raaijmakers $N$, Krug $M$, Regensburger $M$, Zeck $S$ Schinke T, et al: The transcriptional profile of mesenchymal stem cell populations in primary osteoporosis is distinct and shows overexpression of osteogenic inhibitors. PLoS One 7: e45142, 2012.

35. Livak KJ and Schmittgen TD: Analysis of relative gene expression data using real-time quantitative PCR and the 2(-Delta Delta C(T)) method. Methods 25: 402-408, 2001.

36. Huang CJ, Lee CL, Yang SH, Chien CC, Huang CC, Yang RN and Chang CC: Upregulation of the growth arrest-specific-2 in recurrent colorectal cancers, and its susceptibility to chemotherapy in a model cell system. Biochim Biophys Acta 1862: 1345-1353, 2016

37. Chen T, Mao H, Chen C, Wu L, Wang N, Zhao X, Qian J and Xing C: The role and mechanism of $\alpha$-Klotho in the calcification of rat aortic vascular smooth muscle cells. Biomed Res Int 2015: $194362,2015$.

38. Kaji H, Naito J, Sowa H, Sugimoto $T$ and Chihara K: Smad3 differently affects osteoblast differentiation depending upon its differentiation stage. Horm Metab Res 38: 740-745, 2006

39. Sowa H, Kaji H, Yamaguchi T, Sugimoto T and Chihara K: Smad3 promotes alkaline phosphatase activity and mineralization of osteoblastic MC3T3-E1 cells. J Bone Miner Res 17: 1190-1199, 2002

40. Jinnin M, Ihn H and Tamaki K: Characterization of SIS3, a novel specific inhibitor of Smad3, and its effect on transforming growth factor-beta1-induced extracellular matrix expression. Mol Pharmacol 69: 597-607, 2006.

41. Yang P, Lv S, Wang Y, Peng Y, Ye Z, Xia Z, Ding G, Cao X and Crane JL: Preservation of type $H$ vessels and osteoblasts by enhanced preosteoclast platelet-derived growth factor type BB attenuates glucocorticoid-induced osteoporosis in growing mice. Bone 114: 1-13, 2018.

42. Khan MP, Singh AK, Singh AK, Shrivastava P, Tiwari MC, Nagar GK, Bora HK, Parameswaran V, Sanyal S, Bellare JR and Chattopadhyay N: Odanacatib restores trabecular bone of skeletally mature female rabbits with osteopenia but induces brittleness of cortical bone: A comparative study of the investigational drug with PTH, Estrogen, and Alendronate. J Bone Miner Res 31: 615-629, 2016

43. Ishijima M, Sakamoto Y, Yamanaka M, Tokita A, Kitahara K, Kaneko $\mathrm{H}$ and Kurosawa $\mathrm{H}$ : Minimum required vitamin $\mathrm{D}$ level for optimal increase in bone mineral density with alendronate treatment in osteoporotic women. Calcif Tissue Int 85: 398-404, 2009.

44. Park JH, Park KH, Cho S, Choi YS, Seo SK, Lee BS and Park HS Concomitant increase in muscle strength and bone mineral density with decreasing IL-6 levels after combination therapy with alendronate and calcitriol in postmenopausal women Menopause 20: 747-753, 2013.

45. Chou MT, Chang SN, Ke C, Chang HI, Sung ML, Kuo HC and Chen $\mathrm{CN}$ : The proliferation and differentiation of placental-derived multipotent cells into smooth muscle cells on fibrillar collagen. Biomaterials 31: 4367-4375, 2010.

46. Sun J, Li J, Li C and Yu Y: Role of bone morphogenetic protein-2 in osteogenic differentiation of mesenchymal stem cells. Mo Med Rep 12: 4230-4237, 2015.

47. Ho SS, Vollmer NL, Refaat MI, Jeon O, Alsberg E, Lee MA and Leach JK: Bone morphogenetic protein-2 promotes human mesenchymal stem cell survival and resultant bone formation when entrapped in photocrosslinked alginate hydrogels. Adv Healthc Mater 5: 2501-2509, 2016.

48. Baas J, Vestermark M, Jensen T, Bechtold J, Soballe K and Jakobsen T: Topical bisphosphonate augments fixation of bone-grafted hydroxyapatite coated implants, BMP-2 causes resorption-based decrease in bone. Bone 97: 76-82, 2017.

49. Liu Z, Yao X, Yan G, Xu Y, Yan J, Zou W and Wang G: Mediator MED23 cooperates with RUNX2 to drive osteoblast differentiation and bone development. Nat Commun 7: 11149, 2016.
50. Zhao L, Jiang S and Hantash BM: Transforming growth factor beta1 induces osteogenic differentiation of murine bone marrow stromal cells. Tissue Eng Part A 16: 725-733, 2010.

51. Genge BR, Sauer GR, Wu LN, McLean FM and Wuthier RE: Correlation between loss of alkaline phosphatase activity and accumulation of calcium during matrix vesicle-mediated mineralization. J Biol Chem 263: 18513-18519, 1988.

52. Bobryshev YV, Orekhov AN, Sobenin I and Chistiakov DA: Role of bone-type tissue-nonspecific alkaline phosphatase and PHOSPO1 in vascular calcification. Curr Pharm Des 20: 5821-5828, 2014.

53. Sila-Asna M, Bunyaratvej A, Maeda S, Kitaguchi $H$ and Bunyaratavej N: Osteoblast differentiation and bone formation gene expression in strontium-inducing bone marrow mesenchymal stem cell. Kobe J Med Sci 53: 25-35, 2007.

54. Birmingham E, Niebur GL, McHugh PE, Shaw G, Barry FP and McNamara LM: Osteogenic differentiation of mesenchymal stem cells is regulated by osteocyte and osteoblast cells in a simplified bone niche. Eur Cell Mater 23: 13-27, 2012.

55. Yu Y, Yang JL, Chapman-Sheath PJ and Walsh WR: TGF-beta, BMPS, and their signal transducing mediators, Smads, in rat fracture healing. J Biomed Mater Res 60: 392-397, 2002.

56. Chen G, Deng C and Li YP: TGF- $\beta$ and BMP signaling in osteoblast differentiation and bone formation. Int J Biol Sci 8: 272-288, 2012.

57. Bonniaud P, Margetts PJ, Ask K, Flanders K, Gauldie J and Kolb M: TGF-beta and Smad3 signaling link inflammation to chronic fibrogenesis. J Immunol 175: 5390-5395, 2005.

58. Qin W, Chung AC, Huang XR, Meng XM, Hui DS, Yu CM, Sung JJ and Lan HY: TGF- $\beta /$ Smad3 signaling promotes renal fibrosis by inhibiting miR-29. J Am Soc Nephrol 22: 1462-1474, 2011.

59. Dobaczewski M, Bujak M, Li N, Gonzalez-Quesada C, Mendoza LH, Wang XF and Frangogiannis NG: Smad3 signaling critically regulates fibroblast phenotype and function in healing myocardial infarction. Circ Res 107: 418-428, 2010.

60. Flanders KC, Major CD, Arabshahi A, Aburime EE, Okada MH, Fujii M, Blalock TD, Schultz GS, Sowers A, Anzano MA, et al: Interference with transforming growth factor-beta/Smad3 signaling results in accelerated healing of wounds in previously irradiated skin. Am J Pathol 163: 2247-2257, 2003

61. Borton AJ, Frederick JP, Datto MB, Wang XF and Weinstein RS: The loss of Smad3 results in a lower rate of bone formation and osteopenia through dysregulation of osteoblast differentiation and apoptosis. J Bone Miner Res 16: 1754-1764, 2001.

62. Yu FY, Xie CQ, Sun JT, Peng W and Huang XW: Overexpressed miR-145 inhibits osteoclastogenesis in RANKL-induced bone marrow-derived macrophages and ovariectomized mice by regulation of Smad3. Life Sci 202: 11-20, 2018.

63. Tamai $\mathrm{R}$ and Kiyoura $\mathrm{Y}$ : Alendronate augments lipid A-induced IL-1 $\beta$ release and Smad3/NLRP3/ASC-dependent cell death. Life Sci 198: 8-17, 2018.

64. Tamai R, Sugiyama A and Kiyoura Y: Alendronate regulates cytokine production induced by lipid A through nuclear factor- $\mathrm{\kappa B}$ and Smad3 activation in human gingival fibroblasts. J Periodontal Res 46: 13-20, 2011.

65. Rizzoli R, Greenspan SL, Bone G III, Schnitzer TJ, Watts NB, Adami S, Foldes AJ, Roux C, Levine MA, Uebelhart B, et al: Two-year results of once-weekly administration of alendronate $70 \mathrm{mg}$ for the treatment of postmenopausal osteoporosis. J Bone Miner Res 17: 1988-1996, 2002.

66. Uchida S, Taniguchi T, Shimizu T, Kakikawa T, Okuyama K, Okaniwa M, Arizono H, Nagata K, Santora AC, Shiraki M, et al: Therapeutic effects of alendronate $35 \mathrm{mg}$ once weekly and $5 \mathrm{mg}$ once daily in Japanese patients with osteoporosis: A double-blind, randomized study. J Bone Miner Metab 23: 382-388, 2005.

67. Du X, Huang F, Zhang S, Yao Y, Chen Y, Chen Y, Huang H and Bai B: Carboxymethylcellulose with phenolic hydroxyl microcapsules enclosinggene-modified BMSCs for controlled BMP-2 release in vitro. Artif Cells Nanomed Biotechnol 45: 1710-1720, 2017. 